\title{
Cucurbitacin B inhibits tumor angiogenesis by triggering the mitochondrial signaling pathway in endothelial cells
}

\author{
XIAN-MEI PIAO ${ }^{1,2}$, FENG GAO $^{1}$, JIU-XIN ZHU $^{1}$, LI-JUAN WANG $^{3}$, XIN ZHAO $^{1}$, \\ XIN LI ${ }^{1}$, MIAO-MIAO SHENG ${ }^{1}$ and YAN ZHANG ${ }^{1}$ \\ ${ }^{1}$ Department of Pharmacology, College of Pharmacy, Harbin Medical University, The State-Province Key Laboratories of \\ Biomedicine-Pharmaceutics of China, Harbin, Heilongjiang 150086; \\ ${ }^{2}$ Laboratory of Cardiovascular Medicine Research, Harbin Medical University, Ministry of Education, Harbin, \\ Heilongjiang 150081; ${ }^{3}$ Shuangyashan Coal General Hospital, Shuangyashan, Heilongjiang 155100, P.R. China
}

Received November 16, 2016; Accepted February 9, 2018

DOI: $10.3892 /$ ijmm.2018.3647

\begin{abstract}
Cucurbitacin B $(\mathrm{CuB})$, the active component of a traditional Chinese herbal medicine, Pedicellus Melo, has been shown to exhibit antitumor and anti-inflammation effects, but its role in tumor angiogenesis, the key step involved in tumor growth and metastasis, and the involved molecular mechanism are unknown. Tumor angiogenesis is one of the hallmarks of the development in malignant neoplasias and metastasis. Effective targeting of tumor angiogenesis is a key area of interest for cancer therapy. Here, we demonstrated that $\mathrm{CuB}$ significantly inhibited human umbilical vascular endothelial cell (HUVEC) proliferation, migration, tubulogenesis in vitro, and blocked angiogenesis in chick embryo chorioallantoic membrane (CAM) assay in vivo. Furthermore, $\mathrm{CuB}$ induced HUVEC apoptosis and may induce apoptosis by triggering the mitochondrial apoptotic pathway. Finally, we found that $\mathrm{CuB}$ inhibiting angiogenesis was associated with inhibition of the activity of vascular endothelial growth factor receptor 2 (VEGFR2). Our investigations suggested that $\mathrm{CuB}$ was a potential drug candidate for angiogenesis related diseases.
\end{abstract}

\section{Introduction}

Angiogenesis, the formation of new blood vessels from preexisting ones, is a dynamic complex process including cell proliferation, migration and capillary-like tube formation (1). It plays an important role in a wide range of pathophysiological events, including diabetic retinopathy, peripheral vascular

Correspondence to: Professor Yan Zhang, Department of Pharmacology, College of Pharmacy, Harbin Medical University, The State-Province Key Laboratories of Biomedicine-Pharmaceutics of China, 157 Baojian Road, Nangang, Harbin, Heilongjiang 150086, P.R. China

E-mail: 3859833@qq.com

Key words: cucurbitacin B, human umbilical vascular endothelial cell, angiogenesis, vascular endothelial growth factor receptor 2 , apoptosis disease, endometriosis, tissue regeneration, atherosclerosis, obesity, rheumatoid arthritis, and cancer (2-4). Scientists have found that angiogenesis has close relationship with the development of cancer (5-7). Tumor growth needs blood vessels to provide nutrients and discharge metabolic waste (8). Inhibition of angiogenesis has been called the fourth modality of anticancer therapy (9). Vascular endothelial growth factors (VEGFs) are crucial regulators of vascular development during embryogenesis (vasculogenesis) as well as blood vessel formation (angiogenesis) $(10,11)$. Binding of VEGF to vascular endothelial growth factor receptor 1 (VEGFR1) (Flt-1) and VEGFR2 (KDR/Flk-1), two receptors for VEGF with intrinsic tyrosine kinase activity, lead to activation of phospholipase C (PLC), PKC and MAP-kinase. VEGFR2 is the major mediator which plays proangiogenic effect induced by VEGF (12). The inhibitors of VEGFR2 such as sorafenib and sunitinib have been used in clinical treatment of cancer (13-15). Sorafenib inhibited tumor growth by $49 \%$ at the dose of $10 \mathrm{mg} / \mathrm{kg}$, which inhibited the phosphorylation of both ERK and eIF4E, reduced the microvessel area, and induced tumor cell apoptosis in the PLC/PRF/5 xenograft model. Sunitinib is a novel oral small-molecule multitargeted receptor tyrosine kinase inhibitor that has shown direct antitumor activity and antiangiogenic action.

Pedicellus Melo from dry stems of the cucurbitaceous plant Cucumis melo L. Pedicellus Melo is widely used in traditional Chinese medicine to treat digestive system and hepatic diseases, including dyspepsia, jaundice, acute and chronic hepatitis, hepatic cirrhosis and liver cancer. However, its pharmacological mechanism of antitumor effect and the medicinal material basis have not been well studied. Chemical studies have shown that Pedicellus Melo consists of abundant cucurbitacins, which have become a key point of study as promising therapeutic antitumor drugs (16-18). According to the chemical structure, cucurbitacins are divided into twelve categories (19). Cucurbitacin B, cucurbitacin D, cucurbitacin $\mathrm{E}$ and cucurbitacin I have been proven to have antitumor effect through inducing cellular apoptosis $(20,21)$. Cucurbitacin $\mathrm{B}(\mathrm{CuB})$ is the most abundant component in the cucurbitacins of Pedicellus Melo. In vitro experiments also proved the inhibitory effect of $\mathrm{CuB}$ on the growth of human 
cancer cell lines and tumor xenografts including HepG2 cells (22), and SW480 cells (23). CuB has antitumor effect through different mechanisms. The antitumor effect of $\mathrm{CuB}$ on HepG2 cells was due to the induction of cell cycle arrest as well as apoptosis. CuB suppresses non-small-cell lung cancer growth (24), which significantly altered the actin cytoskeletal assembly, induced G2/M cell cycle arrest and mitochondrial apoptosis by targeting cellular thiols. $\mathrm{CuB}$ may inhibit the proliferation of human breast cancer cells through disruption of the microtubule network and downregulation of c-Myc. At present, it is considered that inhibition of angiogenesis is an important means in anticancer therapy. It remains unknown whether $\mathrm{CuB}$ inhibits pathological angiogenesis especially tumor angiogenesis, and its underlying mechanism has not been revealed.

In this study, we found that $\mathrm{CuB}$ inhibited human umbilical vascular endothelial cell (HUVEC) proliferation, migration and tubulogenesis in vitro. We also demonstrated that $\mathrm{CuB}$ suppressed VEGF-induced angiogenesis in chick embryo chorioallantoic membrane (CAM) assay in vivo. Furthermore, our results showed that $\mathrm{CuB}$ induced HUVEC apoptosis, and may induce apoptosis by triggering the mitochondrial apoptotic pathways. Finally, we found that $\mathrm{CuB}$ inhibiting angiogenesis was associated with inhibition of the activity of VEGFR2. Our studies suggested that $\mathrm{CuB}$ is a novel tumor angiogenesis inhibitor and could be a potential drug candidate for angiogenesis related diseases.

\section{Materials and methods}

Cells and reagents. HUVECs and HepG2 cells were obtained from Allcells, LLC (Shanghai, China). CuB (purity 99\%) was purchased from Chengdu Herbpurify Co., Ltd. (Chengdu, China). DMEM/F-12, fetal bovine serum (FBS), was from Gibco (Carlsbad, CA, USA). EGM-2 complete medium was purchased from Lonza Technology (Basel, Switzerland). Twenty-four-well plate with Matrigel was from BD Biosciences (Franklin Lakes, NJ, USA). The Annexin V-FITC/propidium iodide (PI) kit and 3-(4,5-dimethylthiazol-2-yl)-2,5-diphenyltetrazolium bromide (MTT) reagents were purchased from Beyotime Institute of Biotechnology (Shanghai, China). Fertilized chicken eggs were from Harbin Veterinary Research Institute, CAAS (China). VEGF was purchased from PeproTech (Rocky Hill, NJ, USA). Antibodies against VEGFR2 was purchased from Sigma (St. Louis, MO, USA). Antibodies against phosphated-VEGFR2 (Tyr1175) was purchased from Cell Signaling Technology (Beverly, MA, USA). Antibodies for Bcl-2, Bax, caspase-3 and cleaved caspase-3 were purchased from Cell Signaling Technology. $\mathrm{CuB}$ was prepared as a $10 \mathrm{mM}$ stock solution in phosphate-buffered saline (PBS) at $\mathrm{pH}$ 7.4. This stock solution was further diluted to the desired concentration with culture medium. Fresh $\mathrm{CuB}$ stock solution was prepared before use.

Cell culture. HUVEC was cultured in DMEM/F-12 with $10 \%$ FBS, $10 \mathrm{U} / \mathrm{ml}$ penicillin and $10 \mathrm{mg} / \mathrm{ml}$ streptomycin or cultured in complete endothelial cell medium (EGM-2) with grow factors and $2 \%$ FBS in a humidified $5 \% \mathrm{CO}_{2}$ atmosphere at $37^{\circ} \mathrm{C}$. Cells were dissociated with $0.25 \%$ trypsin just before transferring for experiments and counted using a hemocytometer.
Cell viability assay. The effects of extracts isolated from Pedicellus Melo on the proliferation of HUVEC were determined by MTT assay. Briefly, HUVEC was seeded in a 96-well plate in a final volume of $200 \mu \mathrm{l} /$ well of culture medium, at $1 \times 10^{4}$ cells/well $24 \mathrm{~h}$ prior to treatment. The cells were treated with different concentrations of extractions and compounds isolated from Pedicellus Melo. Then $20 \mu 1$ of $5 \mathrm{mg} / \mathrm{ml}$ MTT was added to each well. After $4 \mathrm{~h}$ incubation at $37^{\circ} \mathrm{C}, 150 \mu \mathrm{l}$ dimethyl sulfoxide (DMSO) was added and rocked at room temperature for $15 \mathrm{~min}$. The absorbance was measured at $490 \mathrm{~nm}$. The percentage of the absorbance was calculated against untreated cells. All experiments were repeated three times.

In vitro migration assays. Monolayer HUVEC migration was modified from the method described previously (25). Briefly, HUVECs were allowed to grow to confluence on 6-well plates. After $1 \mathrm{~h}$ of mitomycin C $(2 \mu \mathrm{g} / \mathrm{ml})$ treatment, monolayer cells were scratched with pipette tip and washed three times with PBS. Fresh media with or without $10 \mathrm{ng} / \mathrm{ml}$ VEGF and various concentrations of $\mathrm{CuB}$ were added into the wells. Cells were further cultured for $24 \mathrm{~h}$, and the HUVECs migration in culture was determined by measuring wound distance in cell monolayers. Three different images from each well along the wound were captured by a digital camera under a microscope (x200). Wound distance was measured and analyzed by Image-Pro Plus software.

Capillary-like structure formation assay. HUVECs $\left(1 \times 10^{5}\right)$ were cultured in EGM-2 medium in a Matrigel-precoated 24-well plate at $37^{\circ} \mathrm{C}$. Immediately following cell addition, $\mathrm{CuB}$ was added and the plate tapped gently. The plate was incubated overnight at $37^{\circ} \mathrm{C}$, in $5 \% \mathrm{CO}_{2}$ humidified incubator. After treatment, the medium was aspirated and the plate washed with PBS twice. Then cells were stained with Green Cell Tracker at $10 \mu \mathrm{M}$ dissolved in PBS and incubated for $60 \mathrm{~min}$ at $37^{\circ} \mathrm{C}$, in $5 \% \mathrm{CO}_{2}$ humidified incubator. Each well was washed two times with $1 \mathrm{ml}$ PBS and $200 \mu \mathrm{l}$ PBS was added to each well. Pictures were taken with 10x objective lens. The level of tube formation was quantified by measuring the number of tubes in three randomly chosen fields from each well and was calculated against untreated groups.

In vivo chick embryo chorioallantoic membrane assay. According to a previous method (10), fertilized chick eggs were incubated at $38.5-39^{\circ} \mathrm{C}$ with relative humidity at $65-70 \%$. Five days later, a $1-2 \mathrm{~cm}^{2}$ window was opened with forcep and the shell membrane was removed to expose the chorioallantoic membrane. Sterilized 5-mm diameter filter (Whatman, Maidstone, UK) disks absorbed $\mathrm{CuB}$ or dissolvant alone as control were put onto the avascular area of CAM. Then the window was sealed with filter plastic tape and eggs were incubated for 4 days. The CAM was observed under stereomicroscope and the neovascularization was quantified using Image-Pro Plus software. All animal procedures and experiments were approved by the Institutional Animal Care and Use Committee of Harbin Medical University.

Assessment of apoptosis by Annexin V-FITC/PI staining. Apoptosis analyses of HUVEC cells were carried out by flow cytometry. HUVEC cells were treated with various concentrations of $\mathrm{CuB}$. After the treatment, the cells were 
A

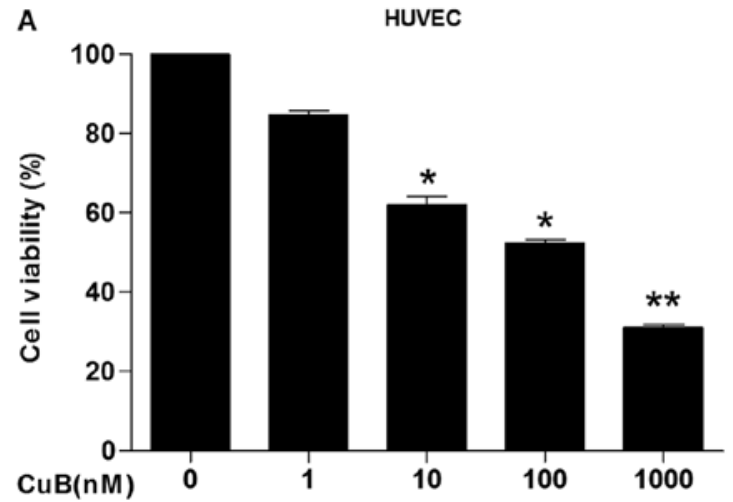

B

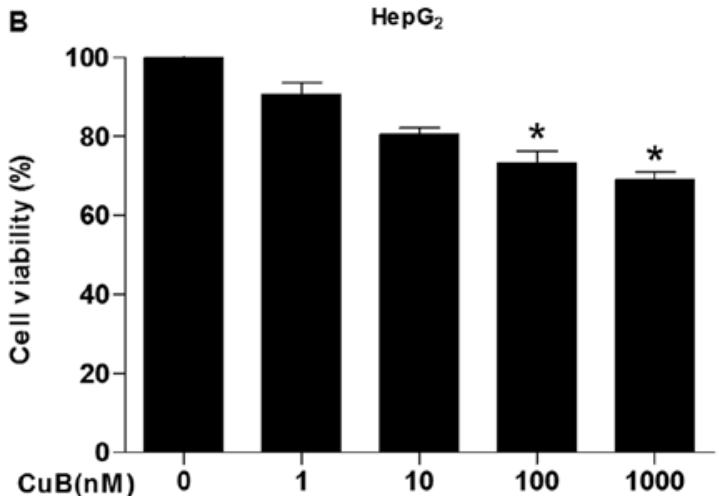

Figure 1. Cucurbitacin B (CuB) inhibits proliferation in human umbilical vascular endothelial cells (HUVEC) and HepG2 cells. HUVEC and HepG2 cells were treated with different concentrations of $\mathrm{CuB}$, then the proliferation was detected by 3-(4,5-dimethylthiazol-2-yl)-2,5-diphenyltetrazolium bromide assay. Untreated cells were $100 \%$. (A) $\mathrm{CuB}$ has strong effect of inhibiting proliferation on HUVEC. ${ }^{*} \mathrm{p}<0.05$ and ${ }^{* *} \mathrm{p}<0.01$. (B) CuB has strong effect of inhibiting proliferation on HepG2 cells. * $\mathrm{p}<0.05$.

harvested by $0.25 \%$ trypsin and washed with PBS and then resuspended in $200 \mu \mathrm{l}$ binding buffer with an addition of $5 \mu \mathrm{l}$ Annexin V-fluorescein isothiocyanate and $10 \mu \mathrm{l} \mathrm{PI}$. The mixture was kept in the dark for $15 \mathrm{~min}$ at $4^{\circ} \mathrm{C}$ and then $300 \mu \mathrm{l}$ of binding buffer was added and analyzed with flow cytometry.

Western blotting. To determine the effects of $\mathrm{CuB}$ on VEGFdependent angiogenesis and HUVEC apoptosis, western blot analysis was performed. Starved HUVECs were treated with $\mathrm{CuB}$ for $2 \mathrm{~h}$ followed by inducing with $100 \mathrm{ng} / \mathrm{ml}$ VEGF165. The apoptosis-related proteins were prepared after treated with indicated concentration of $\mathrm{CuB}$ for $12 \mathrm{~h}$. HUVECs lysates were suspended in RIPA buffer $(10 \mathrm{mmol} / \mathrm{l}$ Tris (pH 7.4), $150 \mathrm{mmol} / \mathrm{l} \mathrm{NaCl}, 1 \%$ Triton $\mathrm{X}-100,1 \%$ deoxycholic acid, 0.1\% SDS, 5 mmol/1 EDTA (pH 8.0), 1 mmol/1 PMSF). Protein concentration was determined using the BCA assay and equalized before loading. Proteins $(80 \mu \mathrm{g})$ were resolved on $12 \%$ sodium dodecyl sulfate-polyacrylamide gel electrophoresis and transferred onto nitrocellulose membranes. The membranes were blocked with 5\% defatted milk in TBS and $0.1 \%$ Tween-20 and probed antibodies overnight at $4^{\circ} \mathrm{C}$. Signals were taken by LI-COR Infrared Imaged Odyssey.

Statistical analysis. Results of cell proliferation, apoptosis and tube formation were expressed as the mean of at least triplicate determinations. Statistical comparisons between drug-treated group and untreated group were performed by SPSS 12.0. Data are presented as mean \pm standard deviations. The bar graphs were prepared using the GraphPad Prism 5. p-values $<0.05$ were considered statistically significant.

\section{Results}

$C u B$ inhibits proliferation in HUVEC. Proliferation of endothelial cells is an important step in the processes of angiogenesis and tumor cells proliferation is required for tumor growth (26). To investigate the inhibitory effects of $\mathrm{CuB}$ on cell proliferation in both endothelial cells and HepG2 cells, we performed the MTS assay with the two types of cells. CuB inhibited the proliferation of HUVEC and HepG2 cells in a dose-dependent manner, and the inhibitory effect of $\mathrm{CuB}$ on the HUVEC was greater than that of HepG2 cells (Fig. 1).
$C u B$ inhibits HUVECs migration. To investigate the potential roles of $\mathrm{CuB}$ in angiogenesis in vitro, we performed migration assays to test the effects of $\mathrm{CuB}$ on HUVECs. Cells in 6-well plates were scratched, washed with phosphate-buffered saline (PBS) and incubated for 8-12 h (Fig. 2). Cells migrated toward the wound regions were analyzed. We found that $\mathrm{CuB}$ inhibited VEGF induced HUVECs migration in woundhealing assay in a dose-dependent manner.

$\mathrm{CuB}$ inhibited tube formation in vitro and angiogenesis in vivo. Endothelial cell tube formation is a key step involved in tumor angiogenesis (27). To study the effect of $\mathrm{CuB}$ on endothelial cell angiogenesis, we added HUVEC with different concentrations of $\mathrm{CuB}$ onto Matrigel and examined the formation of capillary-like structure by HUVECs. Our results showed that the number of tubular capillary-like network on Matrigel was inhibited by $\mathrm{CuB}$ in a dose-dependent manner (Fig. 3A and B). To directly evaluate the effects of $\mathrm{CuB}$ on angiogenesis and vascular development in vivo, we examined the inhibitory effects of $\mathrm{CuB}$ on the chick chorioallantoic membrane to measure the antiangiogenesis property of $\mathrm{CuB}$ in vivo. After 2 days treatment of $\mathrm{CuB}$. The formation of novel blood vessels was obviously blocked by $\mathrm{CuB}(5 \mu \mathrm{g} / \mathrm{disc})$, suggesting that $\mathrm{CuB}$ inhibited chick embryo chorioallantoic membrane angiogenesis, and decreased $62.8 \%$ in branching patterns of the blood vessels (Fig. 3C and D).

$C u B$ induced apoptosis in HUVECs. We further tested the effect of $\mathrm{CuB}$ on cell apoptosis by flow cytometry with PI staining. We evaluated the effect of $\mathrm{CuB}$ on HUVEC apoptosis with Annexin V/PI assay (Fig. 4). The percentage of apoptotic cells in HUVECs was $\sim 50$ and $80 \%$ at the concentration of 100 and 1,000 $\mathrm{nM}$ of $\mathrm{CuB}$, respectively. These data suggested that $\mathrm{CuB}$ can induce apoptosis of endothelial cells.

$\mathrm{CuB}$ triggers the mitochondrial signaling pathway. $\mathrm{CuB}$ inhibited the expression of Bcl-2 and increased the expression of Bax (Fig. 5). On the other hand, we also found that the expression of caspase- 3 was downregulated and cleaved caspase- 3 was upregulated in HUVEC. The above results suggested that $\mathrm{CuB}$ inhibited the mitochondrial signaling pathway. 


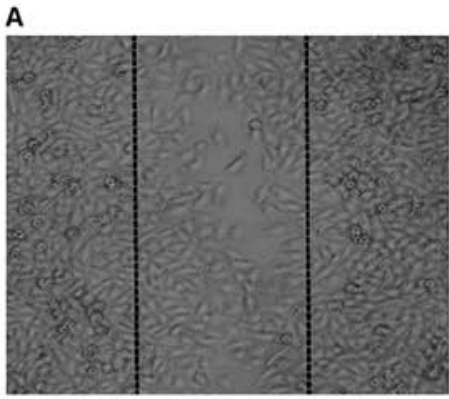

CuB(OnM)

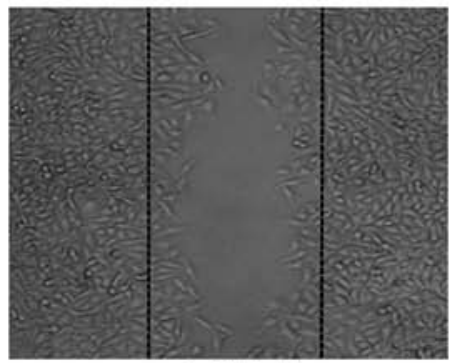

$\mathrm{CuB}(10 \mathrm{nM})$

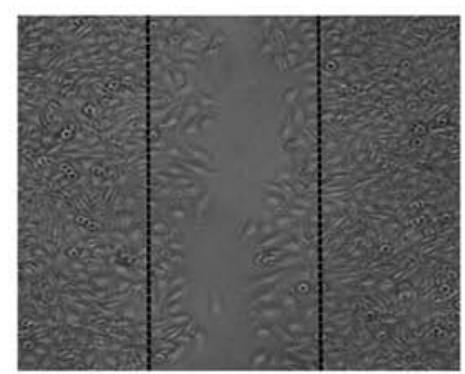

$\mathrm{CuB}(1 \mathrm{n} M)$

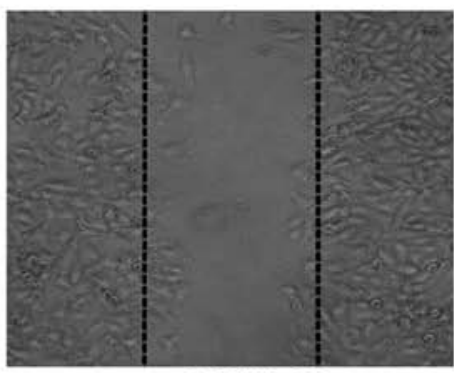

CuB(100nM)
B

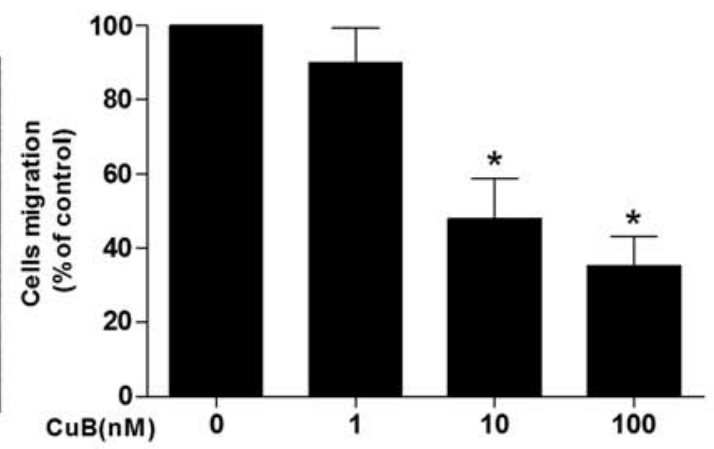

Figure 2. Cucurbitacin B (CuB) inhibits human umbilical vascular endothelial cell (HUVEC) migration. HUVECs were inactivated by $2 \mu \mathrm{g} / \mathrm{ml}$ mitomycin C and induced with $10 \mathrm{ng} / \mathrm{ml}$ vascular endothelial growth factor (VEGF). Dotted lines indicate the area of initial scraping. (A) Representative figure of migration inhibition in HUVECs by $(0,1,10$ and $100 \mathrm{nM}) \mathrm{CuB}$. (B) Migration distance toward the wound regions at $12 \mathrm{~h}$. ${ }^{*} \mathrm{p}<0.05, \mathrm{n}=6$.

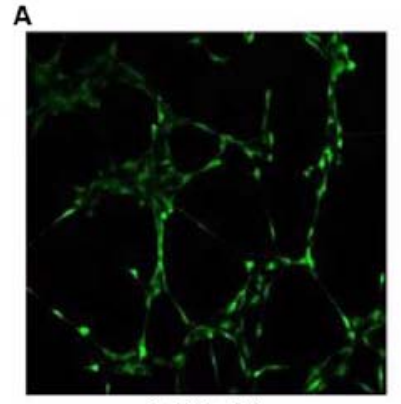

CuB(0 nM)

\section{C}

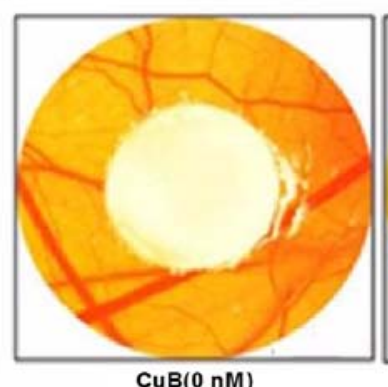

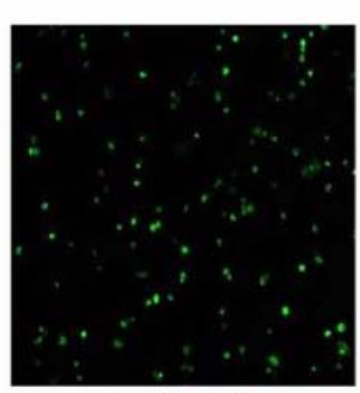

CuB(100 nM)

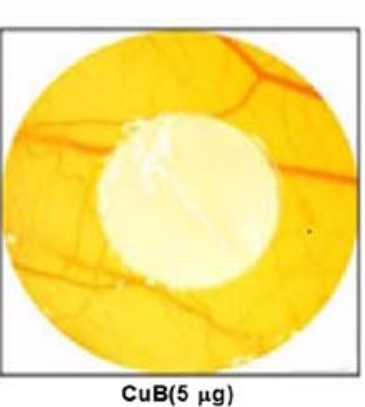

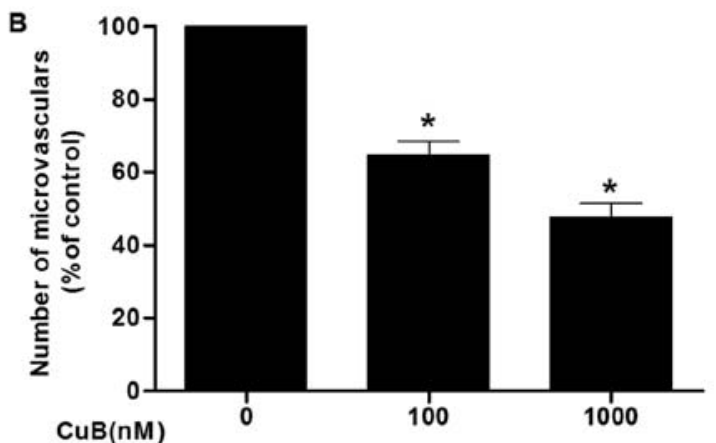

D

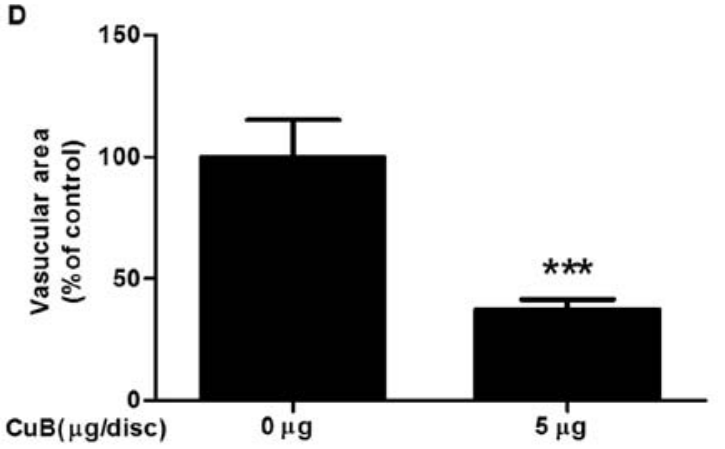

Figure 3. Cucurbitacin B (CuB) inhibits capillary-structure formation of human umbilical vascular endothelial cells (HUVECs) and formation of new blood vessel branches in chorioallantoic membrane (CAM) assay. (A) Representative figures of tube formation inhibition in HUVECs by $100 \mathrm{nM}$ CuB. HUVEC $\left(1 \times 10^{5}\right.$ cells) were seeded in 24-well plate previously coated with Matrigel and treated with CuB for $12 \mathrm{~h}$. (B) The number of tubes after CuB treatment, percent is the proportion in control group. ${ }^{*} \mathrm{p}<0.05, \mathrm{n}=10$. (C) Five micrograms per disc of CuB significantly inhibited microvessel growth in the CAM assay. (D) Statistical analysis of CAM assay. Percent is the proportion of the control group. ${ }^{* * *} \mathrm{p}<0.001, \mathrm{n}=10$.

CUB suppresses VEGF activated VEGFR2 phosphorylation. VEGFR2 is the major receptor of VEGF in angiogenesis, and VEGF/VEGFR2 pathway plays a central role in angiogenesis. To clarify the underlying molecular mechanism of $\mathrm{CuB}$ on angiogenesis, we studied the effects of $\mathrm{CuB}$ on the phosphorylation of VEGFR2 in HUVECs. CuB decreased the VEGF activated VEGFR2 phosphorylation in a dose-dependent manner (Fig. 6). CuB (1000 nM) dramatically inhibited the phosphorylation of VEGFR2, suggesting that $\mathrm{CuB}$ is a potent inhibitor of VEGFR in HUVECs. 

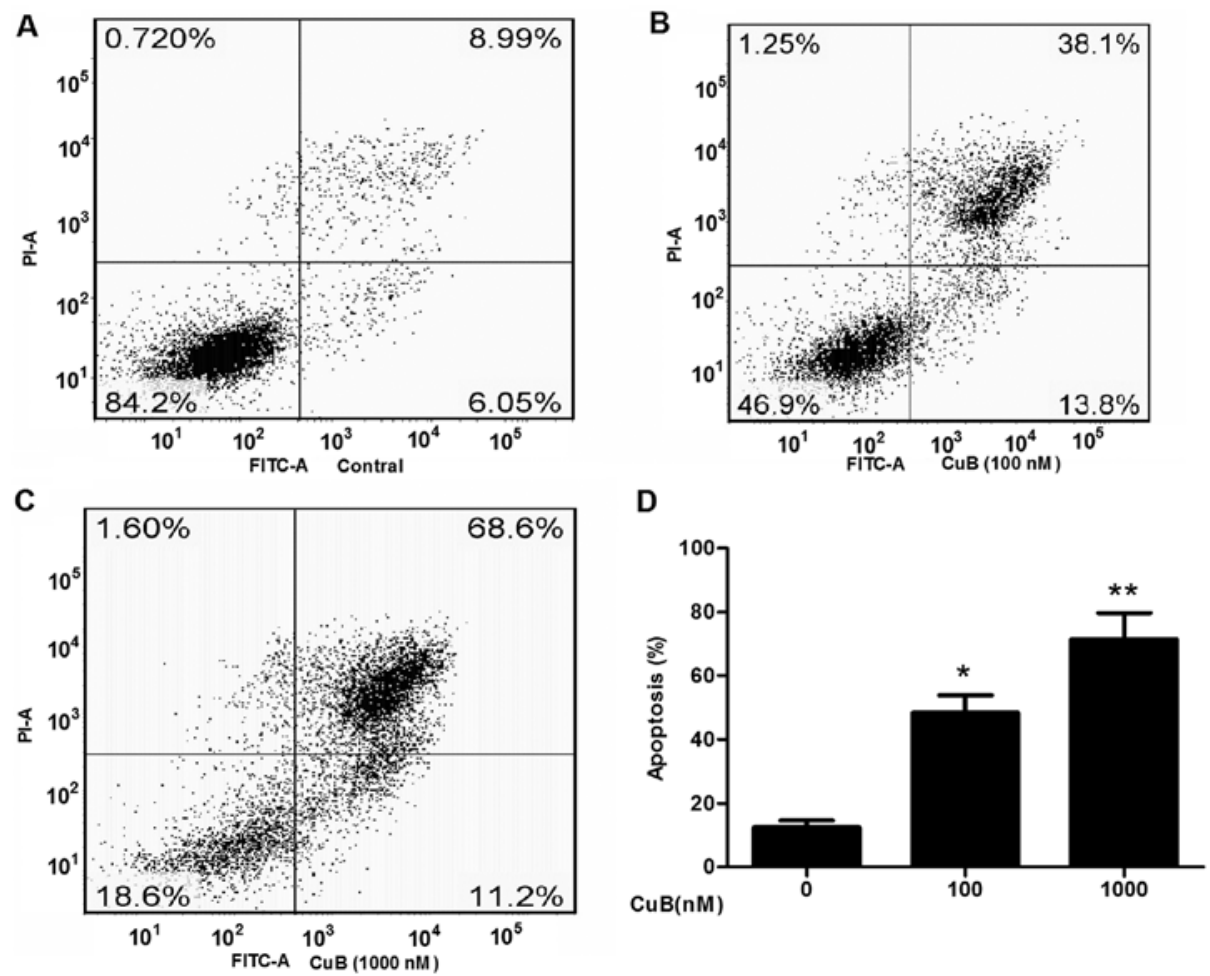

Figure 4. Apoptosis assay of human umbilical vascular endothelial cells (HUVECs) measured by flow cytometry. Cells were treated with different concentrations of cucurbitacin B (CuB) for $12 \mathrm{~h}$ and apoptosis was analyzed by Annexin V-FITC. (A-C) Annexin V-FITC staining is represented on the x-axis and PI staining is represented on the y-axis. Lower right quadrants represent early stage apoptotic cells (Annexin $V$ positive only); upper right quadrants contain late apoptotic and necrotic cells (Annexin V and PI double positive); lower left quadrants represent live cells. (D) The values indicate the percentage of apoptotic cells in each concentration. Results shown are the average of three independent experiments. ${ }^{*} \mathrm{p}<0.05$ and ${ }^{* *} \mathrm{p}<0.01$.
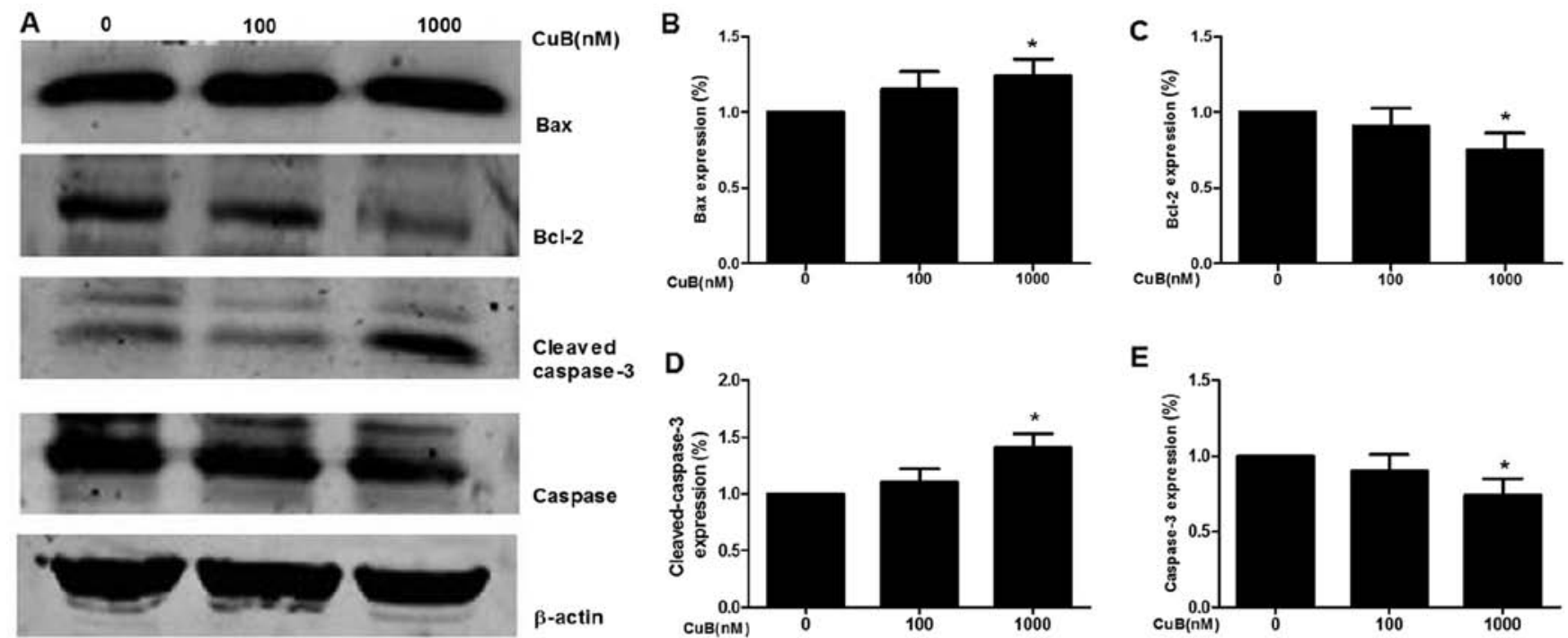

Figure 5. Cucurbitacin $\mathrm{B}(\mathrm{CuB})$ triggeringed the mitochondrial signaling pathway in human umbilical vascular endothelial cells (HUVECs) by western blot analysis. (A) CuB suppressed the expression of Bcl-2 and caspase-3 and increased the expression of Bax and cleaved caspase-3 in a dose-dependent manner. (B-E) Statistical graphs of expression of Bax, Bcl-2, cleaved caspase-3 and caspase-3.

\section{Discussion}

Tumor growth depends on angiogenesis and antiangiogenic therapy is a new target for cancer treatment (28). Identification of angiogenesis inhibitors will benefit drug discovery for tumor and other diseases associated with angiogenesis (29). In this in vivo angiogenesis study, a standard chick embryo
CAM assay of angiogenesis was used. We found that $\mathrm{CuB}$ inhibited CAM angiogenesis in a dose-dependent manner. In angiogenesis study in vitro, CuB suppressed key steps involved in angiogenesis, including proliferation, migration, tubulogenesis, and induced apoptosis in endothelial cells. Further studies illuminated that $\mathrm{CuB}$ induced endothelial cell apoptosis via suppressing the mitochondrial pathway. 

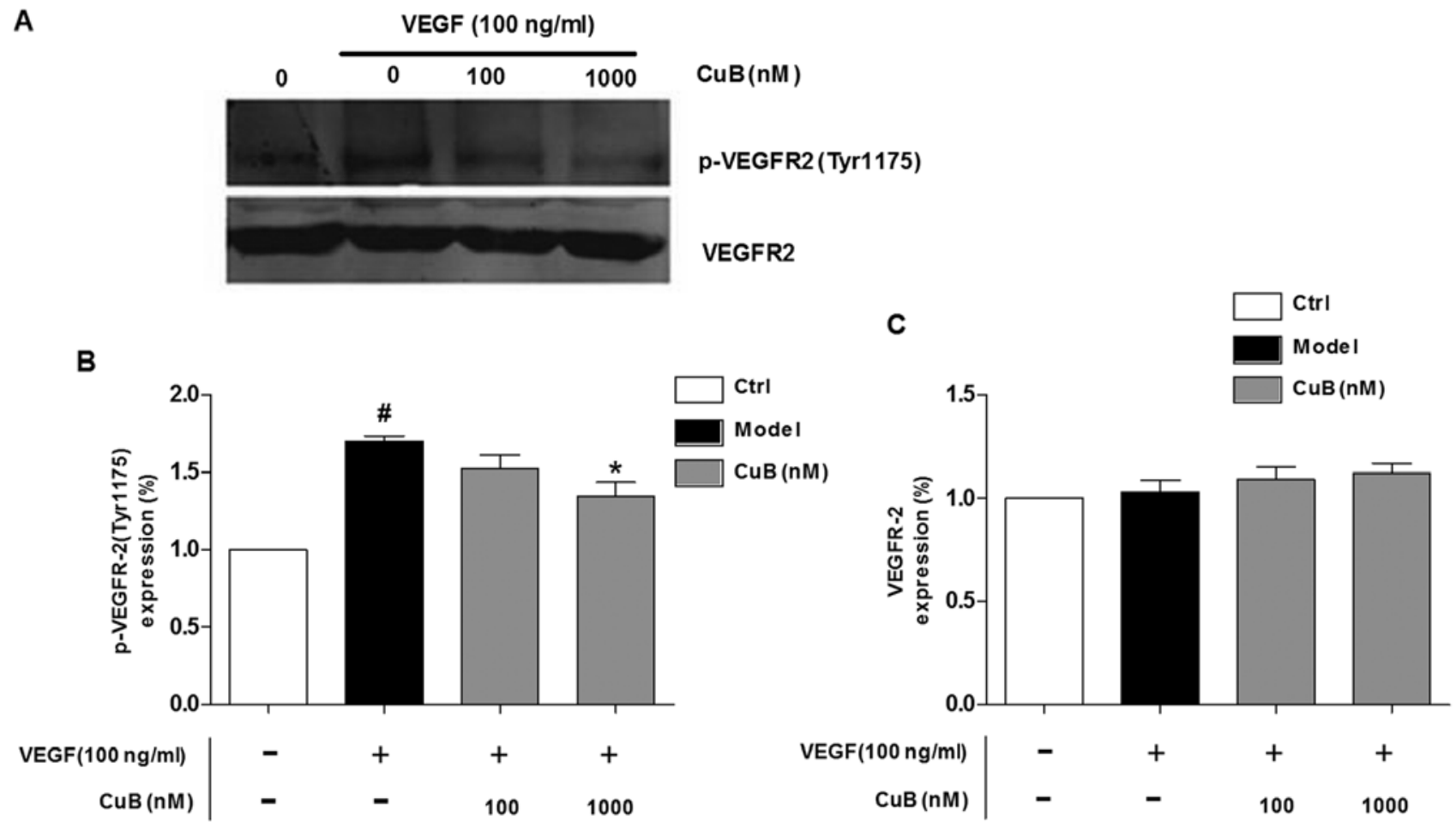

Figure 6. Effects of cucurbitacin B (CuB) on the expression of pTyr1175-vascular endothelial growth factor receptor 2 (VEGFR2) and VEGFR2 in human umbilical vascular endothelial cells (HUVECs). (A) CuB suppressed VEGF-induced phosphorylation activity of VEGFR2 in phosphorylation sites Tyr1175. HUVECs were treated by VEGF alone or combined with different concentrations of CuB for $2 \mathrm{~h}$. (B) Statistical graph of pTyr1175-VEGFR2. Data are expressed as mean $\pm \mathrm{SD},{ }^{\sharp} \mathrm{p}<0.05$ vs. Ctrl; ${ }^{*} \mathrm{p}<0.05$ vs. model, $\mathrm{n}=5$. (C) Statistical graph of VEGFR2. Data are expressed as mean $\pm \mathrm{SD}$.

Pedicellus Melo L. belongs to the Cucurbitaceae family which consists of various cucurbitacins used as a natural medicine in China for centuries. There is increasing evidence showing that cucurbitacins not only inhibited cancer cell proliferation and induced cell apoptosis, but also showed the synergistic effect with known chemotherapeutic agents, such as doxorubicin and gemcitabine. The reported molecular mechanisms of action of cucurbitacins in human cancer cells not only inhibited signal transducers and activators of transcription-3 (STAT3), but also affected other signaling pathways, such as the MAPK pathway (30). Cucurbitacins as potential anticancer drugs have attracted the attention of the drug industry. It has been reported that cucurbitacins especially $\mathrm{CuB}$ is widely utilized for antitumor activity in traditional Chinese medicine $(31,32)$. In this study, we demonstrated that $\mathrm{CuB}$ significantly inhibited proliferation in HUVEC. Interestingly, $\mathrm{CuB}$ inhibited much stronger proliferation in endothelial cells than that in HepG2 cells. These results suggested whether $\mathrm{CuB}$ inhibited tumor growth mainly through targeting endothelial cells other than tumor cells. With the development of the pharmacological mechanism in cucurbitacins, cucurbitacin $\mathrm{E}$ has been proven to be an anti-angiogenic agent by inhibiting VEGFR2-mediated Jak-STAT3 and mitogen-activated protein kinases signaling pathways. However, whether the same kind of compound as $\mathrm{CuB}$, can also inhibit tumor angiogenesis is still unknown. Whether the anti-angiogenisis will be a new therapeutic target for cucurbitacins need to be further studied.

Angiogenesis is a very complex process, it is the result of the interaction of different factors in time and space. Angiogenesis begins with the degradation of the basement membrane by activated endothelial cells that will migrate and proliferate, leading to the formation of solid endothelial cell sprouts in the stromal space. The activation and proliferation of endothelial cells is the first step in this process. In this study, we demonstrated that $\mathrm{CuB}$ significantly inhibited proliferation in HUVEC. The migration and new vessel formation of endothelial cells is also the key steps in the angiogenesis. In our results, $\mathrm{CuB}$ obviously inhibited the two steps in angiogenesis. The chick CAM assay is a typical and widely used experiment in angiogenesis study. In this in vivo experiment, the formation of novel blood vessels was obviously blocked by $\mathrm{CuB}$. It suggested that $\mathrm{CuB}$ may suppress angiogenesis in vivo and in vitro.

In the process of angiogenesis, endothelial cell apoptosis is an important factor for the inhibition of angiogenesis. There is a dynamic balance between the formation of new blood vessels and apoptosis, if it can promote the apoptosis, angiogenesis will be inhibited. Increasing number of studies have shown that mitochondria played a crucial role in cellular apoptotic pathway (33). Bcl-2 protein family plays an essential role in the regulation of mitochondrial apoptosis pathway (34). The pro-apoptotic Bcl-2 family protein Bax has been shown to be required for the disruption of mitochondrial of cancer cells, whereas the antiapoptotic $\mathrm{Bcl}-2$ can prevent cell death by interfering with the activation of Bax (35). In the present study, we found that the expression level of $\mathrm{Bcl}-2$ was suppressed and the expression of Bax was upregulated in HUVEC. To further characterize the molecular mechanisms of $\mathrm{CuB}$-induced apoptotic pathways, we examined the expression of caspase- 3 and cleaved caspase-3. Compared with control group, the $\mathrm{CuB}$ could downregulate the expression of caspase- 3 and upregulate the expression of cleaved caspase- 3 . Thus, we concluded that 
$\mathrm{CuB}$ may induce apoptosis in endothelia cells by triggering the mitochondrial apoptotic pathways.

VEGFs are critical regulators of vasculogenesis and angiogenesis by binding to their cognate receptor. Among the three receptors (VEGFR1,2,3), VEGFR2 is the main receptor of VEGF involved in angiogenesis (36). Activation of the VEGF signaling pathway triggers a network of signaling processes that promote endothelial cell growth, migration and tube formation. In this study, we found that the activity of VEGFR2 was inhibited by $\mathrm{CuB}$ in a dose-dependent manner. This result showed that $\mathrm{CuB}$ inhibited VEGF mediated angiogenesis through VEGFR2 inhibition.

Angiogenesis is a complex multistep process. Inhibition of any step of these processes may result in the damage of angiogenesis and can serve as a potential antitumor therapy. We found $\mathrm{CuB}$ inhibited angiogenesis effects of tumors by triggering the mitochondrial apoptotic pathways in endothelia cells. Shukla et al also reported that $\mathrm{CuB}$ inhibited metastasis and angiogenesis of non-small cell lung cancer which was related with the downregulation of $\mathrm{Wnt} / \beta$-catenin signaling axis (37). This shows that $\mathrm{CuB}$ inhibited angiogenesis possibly through different mechanisms. The present study suggests that $\mathrm{CuB}$ as a VEGFR2 inhibitor is a potential drug candidate for diseases associated with pathological angiogenesis.

In conclusion, our studies showed that $\mathrm{CuB}$ significantly inhibited human umbilical vascular endothelial cell (HUVEC) proliferation, migration, tubulogenesis in vitro, and blocked angiogenesis in chick embryo chorioallantoic membrane assay in vivo. Furthermore, $\mathrm{CuB}$ induced HUVEC apoptosis and may induce apoptosis by triggering the mitochondrial apoptotic pathway. Finally, we found that $\mathrm{CuB}$ inhibiting angiogenesis was associated with inhibition of the activity of VEGFR2. Our new finding of $\mathrm{CuB}$ in angiogenesis suggested a novel role of $\mathrm{CuB}$ as an antiangiogenesis agent.

\section{Acknowledgements}

Not applicable.

\section{Funding}

This study was supported by the Heilongjiang Postdoctoral fund, Heilongjiang Province Science Foundation for Youths (no. QC2013C081) the funding from Key Laboratory of Cardiovascular Medicine Research (Harbin Medical University), the National Natural Science Foundation of China (no. 81703760) and the Central Guide Local Project of Science and Technology Development (grant no. ZY16A07).

\section{Availability of data and material}

The datasets used and/or analyzed during the current study are available from the corresponding author on reasonable request.

\section{Authors' contributions}

XMP was mainly responsible for the design and writing of the article, and performed some experimental operations. FG performed the cell viability assay and capillary-like structure formation assay. JXZ and LJW performed the western blot- ting experiment. XZ was a major contributor in data analysis. $\mathrm{XL}$ was in charge of making diagrams. MMS participated in other in vitro experiments. YZ was mainly responsible for the conception and revision of the article. All authors read and approved the final manuscript.

\section{Ethics approval and consent to participate}

All animal procedures and experiments were approved by the Institutional Animal Care and Use Committee of Harbin Medical University.

\section{Consent for publication}

Not applicable.

\section{Competing interests}

The authors declare that they have no competing interests.

\section{References}

1. Zhang K, Lu J, Mori T, Smith-Powell L, Synold TW, Chen S and Wen W: Baicalin increases VEGF expression and angiogenesis by activating the ERR $\{\alpha\} / \mathrm{PGC}-1\{\alpha\}$ pathway. Cardiovasc Res 89: 426-435, 2011.

2. Carmeliet P: Angiogenesis in life, disease and medicine. Nature 438: 932-936, 2005.

3. Folkman J: Angiogenesis: An organizing principle for drug discovery? Nat Rev Drug Discov 6: 273-286, 2007.

4. Cao Y: Adipose tissue angiogenesis as a therapeutic target for obesity and metabolic diseases. Nat Rev Drug Discov 9: 107-115, 2010.

5. Li WW, Li VW, Hutnik M and Chiou AS: Tumor angiogenesis as a target for dietary cancer prevention. J Oncol 2012: 879623, 2012.

6. Folkman J: Angiogenesis in cancer, vascular, rheumatoid and other disease. Nat Med 1: 27-31, 1995.

7. Grothey A and Galanis E: Targeting angiogenesis: Progress with anti-VEGF treatment with large molecules. Nat Rev Clin Oncol 6: 507-518, 2009.

8. Tammali R, Reddy AB, Srivastava SK and Ramana KV: Inhibition of aldose reductase prevents angiogenesis in vitro and in vivo. Angiogenesis 14: 209-221, 2011.

9. Folkman J: Endogenous angiogenesis inhibitors. APMIS 112: 496-507, 2004.

10. Yi ZF, Cho SG, Zhao H, Wu YY, Luo J, Li D, Yi T, Xu X, Wu Z and Liu M: A novel peptide from human apolipoprotein(a) inhibits angiogenesis and tumor growth by targeting c-Src phosphorylation in VEGF-induced human umbilical endothelial cells. Int J Cancer 124: 843-852, 2009.

11. Yi T, Cho SG, Yi Z, Pang X, Rodriguez M, Wang Y, Sethi G, Aggarwal BB and Liu M: Thymoquinone inhibits tumor angiogenesis and tumor growth through suppressing AKT and extracellular signal-regulated kinase signaling pathways. Mol Cancer Ther 7: 1789-1796, 2008.

12. Tang N, Wang L, Esko J, Giordano FJ, Huang Y, Gerber HP, Ferrara N and Johnson RS: Loss of HIF-1alpha in endothelial cells disrupts a hypoxia-driven VEGF autocrine loop necessary for tumorigenesis. Cancer Cell 6: 485-495, 2004.

13. Liu L, Cao Y, Chen C, Zhang X, McNabola A, Wilkie D, Wilhelm S, Lynch $\mathrm{M}$ and Carter C: Sorafenib blocks the RAF/MEK/ERK pathway, inhibits tumor angiogenesis, and induces tumor cell apoptosis in hepatocellular carcinoma model PLC/PRF/5. Cancer Res 66: 11851-11858, 2006.

14. Kane RC, Farrell AT, Saber H, Tang S, Williams G, Jee JM, Liang C, Booth B, Chidambaram N, Morse D, et al: Sorafenib for the treatment of advanced renal cell carcinoma. Clin Cancer Res 12: 7271-7278, 2006.

15. Cabebe E and Wakelee H: Sunitinib: A newly approved smallmolecule inhibitor of angiogenesis. Drugs Today (Barc) 42: 387-398, 2006 
16. Alghasham AA: Cucurbitacins - a promising target for cancer therapy. Int J Health Sci (Qassim) 7: 77-89, 2013.

17. Molavi O, Ma Z, Mahmud A, Alshamsan A, Samuel J, Lai R. Kwon GS and Lavasanifar A: Polymeric micelles for the solubilization and delivery of STAT3 inhibitor cucurbitacins in solid tumors. Int J Pharm 347: 118-127, 2008.

18. Bishayee A, Ahmed S, Brankov N and Perloff M: Triterpenoids as potential agents for the chemoprevention and therapy of breast cancer. Front Biosci (Landmark Ed) 16: 980-996, 2011

19. Chen JC, Chiu MH, Nie RL, Cordell GA and Qiu SX: Cucurbitacins and cucurbitane glycosides: Structures and biological activities. Nat Prod Rep 22: 386-399, 2005.

20. Duangmano S, Sae-Lim P, Suksamrarn A, Domann FE and Patmasiriwat P: Cucurbitacin B inhibits human breast cancer cell proliferation through disruption of microtubule polymerization and nucleophosmin/B23 translocation. BMC Complement Altern Med 12: 185, 2012.

21. Dakeng S, Duangmano S, Jiratchariyakul W, U-Pratya Y, Bögler $\mathrm{O}$ and Patmasiriwat P: Inhibition of Wnt signaling by cucurbitacin B in breast cancer cells: Reduction of Wnt-associated proteins and reduced translocation of galectin-3-mediated $\beta$-catenin to the nucleus. J Cell Biochem 113: 49-60, 2012.

22. Liu T, Zhang M, Zhang H, Sun C and Deng Y: Inhibitory effects of cucurbitacin B on laryngeal squamous cell carcinoma. Eur Arch Otorhinolaryngol 265: 1225-1232, 2008.

23. Yasuda S, Yogosawa S, Izutani Y, Nakamura Y, Watanabe $\mathrm{H}$ and Sakai T: Cucurbitacin B induces G2 arrest and apoptosis via a reactive oxygen species-dependent mechanism in human colon adenocarcinoma SW480 cells. Mol Nutr Food Res 54: 559-565, 2010.

24. Kausar H, Munagala R, Bansal SS, Aqil F, Vadhanam MV and Gupta RC: Cucurbitacin B potently suppresses non-small-cell lung cancer growth: Identification of intracellular thiols as critical targets. Cancer Lett 332: 35-45, 2013.

25. Luo D, Luo Y, He Y, Zhang H, Zhang R, Li X, Dobrucki WL, Sinusas AJ, Sessa WC and Min W: Differential functions of tumor necrosis factor receptor 1 and 2 signaling in ischemiamediated arteriogenesis and angiogenesis. Am J Pathol 169: 1886-1898, 2006.

26. Lynch CN, Wang YC, Lund JK, Chen YW, Leal JA and Wiley SR: TWEAK induces angiogenesis and proliferation of endothelial cells. J Biol Chem 274: 8455-8459, 1999.
27. Kaneko T, Nagata I, Miyamoto S, Kubo H, Kikuchi H, Fujisato T and Ikada Y: Effects of nicardipine on tube formation of bovine vascular endothelial cells in vitro. Stroke 23: 1637-1642, 1992.

28. Chen Z and Han ZC: STAT3: A critical transcription activator in angiogenesis. Med Res Rev 28: 185-200, 2008.

29. Zhang X, Song Y, Wu Y, Dong Y, Lai L, Zhang J, Lu B, Dai F, He L, Liu M, et al: Indirubin inhibits tumor growth by antitumor angiogenesis via blocking VEGFR2-mediated JAK/STAT3 signaling in endothelial cell. Int J Cancer 129: 2502-2511, 2011.

30. Boykin C, Zhang G, Chen YH, Zhang RW, Fan XE, Yang WM and Lu Q: Cucurbitacin IIa: A novel class of anticancer drug inducing non-reversible actin aggregation and inhibiting survivin independent of JAK2/STAT3 phosphorylation. Br J Cancer 104: 781-789, 2011.

31. Chan KT, Li K, Liu SL, Chu KH, Toh M and Xie WD Cucurbitacin B inhibits STAT3 and the Raf/MEK/ERK pathway in leukemia cell line K562. Cancer Lett 289: 46-52, 2010.

32. Gupta P and Srivastava SK: Inhibition of Integrin-HER2 signaling by cucurbitacin B leads to in vitro and in vivo breast tumor growth suppression. Oncotarget 5: 1812-1828, 2014.

33. Onishi Y, Kawamoto T, Ueha T, Kishimoto K, Hara H, Fukase N, Toda M, Harada R, Minoda M, Sakai Y, et al: Transcutaneous application of carbon dioxide (CO2) induces mitochondrial apoptosis in human malignant fibrous histiocytoma in vivo. PLoS One 7: e49189, 2012.

34. Fesik SW: Promoting apoptosis as a strategy for cancer drug discovery. Nat Rev Cancer 5: 876-885, 2005.

35. Jiang L, Luo M, Liu D, Chen B, Zhang W, Mai L, Zeng J, Huang N, Huang Y, Mo X, et al: BAD overexpression inhibits cell growth and induces apoptosis via mitochondrial-dependent pathway in non-small cell lung cancer. Cancer Cell Int 13: 53, 2013.

36. Olsson AK, Dimberg A, Kreuger J and Claesson-Welsh L: VEGF receptor signalling - in control of vascular function. Nat Rev Mol Cell Biol 7: 359-371, 2006.

37. Shukla S, Sinha S, Khan S, Kumar S, Singh K, Mitra K, Maurya R and Meeran SM: Cucurbitacin B inhibits the stemness and metastatic abilities of NSCLC via downregulation of canonical Wnt/ß-catenin signaling axis. Sci Rep 6: 21860, 2016. 